\title{
Cancer Incidence and Mortality in Chronic Dialysis Population: A Multicenter Cohort Study
}

\author{
Chi Yuen Cheung a Gary C.W. Chan ${ }^{\mathrm{b}}$ Siu Kim Chan ${ }^{\mathrm{c}}$ Flora Ng ${ }^{\mathrm{d}}$ \\ Man Fai Lam ${ }^{b}$ Sunny S.H. Wong ${ }^{c}$ Wai Leung Chak ${ }^{a} K^{2}$ Foon Chau ${ }^{a}$ \\ Sing Leung Lui ${ }^{d}$ Wai Kei Lo ${ }^{d}$ Sydney C.W. Tang ${ }^{b}$ \\ ${ }^{a}$ Renal Unit, Department of Medicine, Queen Elizabeth Hospital, ${ }^{b}$ Division of Nephrology, Department of \\ Medicine, The University of Hong Kong, Queen Mary Hospital, 'Department of Medicine and Geriatrics, \\ United Christian Hospital, 'Dr. Lee lu Cheung Memorial Renal Research Center, Department of Medicine, \\ Tung Wah Hospital, Hong Kong, SAR, China
}

\section{Key Words}

Cancer · Dialysis · Incidence $\cdot$ Mortality

\begin{abstract}
Background: Different studies in the past have shown that the risk of cancer development is increased in chronic dialysis patients. However, data concerning the cancer risk in Asian dialysis patients was scarce. More importantly, there was lack of information about the cancer-specific mortality in dialysis patients. Methods: A multicenter retrospective cohort study of 6,254 patients who started either chronic peritoneal dialysis or hemodialysis between 1994 and 2014 in 4 renal units in Hong Kong. Patterns of cancer incidence and mortality in our dialysis patients were compared with those of the general population using standardized incidence ratios (SIRs) and standardized mortality ratios (SMRs) respectively. Results: With 14,887 person-years of follow-up, 220 cancers were recorded. The SIR of all cancers was 1.44 (95\% Cl 1.26-1.65). A trend of an increased SIR was observed in young patients and within the first year of dialysis. Colorectum was the most common site of cancer (20\%) while kidney cancer carried the highest risk (SIR 12.28, 95\% Cl 8.44-17.08). The SMR of all cancers was 0.91 (95\% Cl $0.72-1.13)$ and only
\end{abstract}

\section{KARGER}

E-Mail karger@karger.com

www.karger.com/ajn kidney cancer had higher cancer mortality risk (SMR 4.92, $95 \% \mathrm{Cl} 1.80-10.70$ ). SMR was highest in young patients and then decreased with age. Conclusions: The incidence of cancers in our chronic dialysis patients was elevated. Our findings of substantially increased risks in young patients, particularly in relation to kidney cancer, suggest that we can adopt a more individualized approach to cancer screening in chronic dialysis patients.

(c) 2016 S. Karger AG, Basel

\section{Introduction}

Although kidney transplantation is considered to be the most definite treatment for patients suffering from end stage kidney disease (ESKD), dialysis is still the commonest treatment for these patients. Different studies in the past have shown that the risk of cancer development is increased in ESKD patients, whether on dialysis or not, when compared with the general popula-

Dr. Chi Yuen Cheung

Department of Medicine

Queen Elizabeth Hospital, 30 Gascoigne Road

Kowloon, Hong Kong, SAR (China)

E-Mail simoncycheung@gmail.com

Prof. Sydney C.W. Tang

Department of Medicine

Queen Mary Hospital, The University of Hong Kong

102 Pokfulam Road, Hong Kong, SAR (China)

E-Mail scwtang@hku.hk 
tion [1-8]. The relative risk of cancer is increased when the glomerular filtration rate (GFR) is $<60 \mathrm{ml} / \mathrm{min}$, with men having a $29 \%$ increase in cancer risk for every 10 $\mathrm{ml}$ reduction in GFR [9]. In addition, mortality from cancers was also higher in dialysis patients than in the general population $[10,11]$. The postulated pathogenic mechanisms for cancer development in this group of patients include: altered DNA repair, impaired immune system function, decreased antioxidant defence, chronic infection and inflammation and accumulation of carcinogenic compounds [12]. In addition, the underlying disease leading to renal failure and the development of acquired cystic disease may also predispose to cancer development in patients with ESKD [13]. In a large international collaborative study, higher risks were observed for cancers of the urinary bladder, kidney, thyroid and other endocrine tumors, with approximately $13-25 \%$ of all cancers occurring in the kidney or urinary bladder [3].

However, these studies were mainly focused on nonAsian ethnicities and there is paucity of data in Asian chronic dialysis patients. In addition, the pattern and frequency of various cancers may vary widely even among dialysis patients from different Asian countries [14-17]. Chronic dialysis patients in Korea had a higher frequency of gastrointestinal tract and urinary tract cancers [14] while the patients in Japan had a higher frequency of gynecological and urinary tract cancers [15]. In Singapore, the 3 sites with highest elevated cancer risks among dialysis patients were kidney, tongue and multiple myeloma [16]. A study in Taiwan several years ago showed that urinary bladder and liver cancers were the most common cancers in their chronic dialysis patients [17]. However, it is not clear whether ethnicity alone can explain all the discrepancies between the Taiwanese study and the international collaborative study as this was the only study conducted in the Chinese population so far $[3,17]$. It would be interesting to find out if the Chinese dialysis patients in other areas also have similar pattern of cancers. Moreover, information concerning the cancer mortality in dialysis patients is lacking. Here, we conducted a study to examine the risk and outcome of various types of cancers among the Chinese chronic dialysis patients who had not undergone transplantation.

\section{Subjects and Methods}

This is a multicenter retrospective cohort study of all incident patients suffering from ESKD who started either peritoneal dialysis (PD) or hemodialysis (HD) in 4 large renal units in Hong
Kong, including Queen Elizabeth Hospital, Queen Mary Hospital, Tung Wah Hospital and United Christian Hospital within the period between January 1, 1994 and September 30, 2014. All cancer events were collected from the Hong Kong Renal Registry database. The Renal Registry is a direct, online, computerized registry system developed by the Central Renal Committee, Hospital Authority (HA), to collect data of all patients who received renal replacement therapy (RRT) under HA [18]. The registry accounts for $90-95 \%$ of all RRT patients in our territory. The data are entered directly by the doctors or nurses of the individual renal unit and each unit can access their own patient data. All cancers were diagnosed and verified with histology and other relevant information, such as radiological imaging, and coded according to the 10th World Health Organization International Classification of Disease-10. Patients who had previous history of cancers, acquired immune deficiency syndrome or kidney transplantation prior to dialysis were excluded from analysis. Only the first cancer diagnosis after dialysis therapy initiation was included. Patients with multiple cancer sites diagnosed simultaneously were included in each site-specific analysis. The basic demographic and clinical data including gender, age, underlying kidney diseases, types of cancers, interval from the initiation of dialysis to the development of cancers, interval from diagnosis of cancers to death were extracted from patients' medical record. For purposes of the analyses and defining years of follow-up, patients were censored at the time of death, diagnosis of malignancy, at the time of kidney transplantation, the last reported contact or December 31, 2014. This study was approved by the research ethical committee of all 4 hospitals.

SPSS (SPSS 21.0, IBM, USA) was used to perform the statistical analyses. Continuous data were expressed as means $\pm \mathrm{SD}$; categorical data were expressed as percentages. The standardized incidence ratio (SIR) and frequency of various cancers in dialysis patients were measured. In order to compare the cancer risk between the chronic dialysis patients and the general population, the number of person-years at risk of malignancy was first calculated. SIR was then calculated as the number of observed cancer cases among the chronic dialysis patients divided by the expected number of cancer cases. The expected number of cases was based on the person-years at risk and the age-adjusted cancer incidence rates in the general population in Hong Kong, which were obtained from the Hong Kong Cancer Registry, provided by the HA [19]. Through the Hong Kong Cancer Registry, the general population incidence and mortality rates by 5 -year age group and gender for each year from 1983 to 2012 could be obtained for all cancer sites; rates for 2012 were used for later years. The $95 \%$ CIs of the SIRs could be calculated by assuming that the observed cancers follow a Poisson distribution. In addition, we also compared the cancer mortality between the chronic dialysis patients and the general population. A cancer death was defined as primary cause of death due to cancer. The observed rates of cancer death were calculated as the number of deaths from cancer per 100,000 patient-years among the dialysis patients. The expected rates of cancer death were the number of deaths from cancer per 100,000 patient-years among the general population, which were also obtained from the Hong Kong Cancer Registry. The standardized mortality ratio (SMR) was the observed rates/expected rates and the $95 \%$ CIs were calculated by assuming that the observed mortality follow a Poisson distribution. A p value of $<0.05$ was defined as statistically significant in this study. 
Table 1. Distribution and clinical characteristics of malignancies after dialysis

\begin{tabular}{|c|c|c|c|c|c|}
\hline & Number & Percentage & $\begin{array}{l}\text { Age at dialysis, } \\
\text { years }\end{array}$ & $\begin{array}{l}\text { Age at diagnosis } \\
\text { of cancers, years }\end{array}$ & $\begin{array}{l}\text { Duration between dialysis } \\
\text { and cancers, months }\end{array}$ \\
\hline Colorectal CA & 44 & 20.0 & $65.1 \pm 11 / 7$ & $68.8 \pm 10.6$ & $44(2-143)$ \\
\hline Renal cell CA & 35 & 15.9 & $50.5 \pm 12.2$ & $57.9 \pm 10.2$ & $89(2-232)$ \\
\hline Lung CA & 30 & 13.6 & $66.9 \pm 10.6$ & $69.9 \pm 8.8$ & $37(2-279)$ \\
\hline Breast CA & 18 & 8.2 & $54.3 \pm 14.0$ & $58.4 \pm 11.9$ & $49(2-214)$ \\
\hline Hepatocellular CA & 14 & 6.4 & $53.1 \pm 17.2$ & $59.8 \pm 13.1$ & $80(5-327)$ \\
\hline Bladder CA & 9 & 4.1 & $64.0 \pm 13.0$ & $68.8 \pm 11.0$ & $58(9-154)$ \\
\hline NMSC & 8 & 3.6 & $64.5 \pm 10.9$ & $68.1 \pm 9.4$ & $43(14-117)$ \\
\hline Stomach CA & 8 & 3.6 & $70.0 \pm 10.9$ & $72.5 \pm 12.1$ & $33(3-84)$ \\
\hline NHL & 7 & 3.2 & $64.0 \pm 9.0$ & $69.3 \pm 9.5$ & $65(2-136)$ \\
\hline Cervical CA & 7 & 3.2 & $58.3 \pm 8.8$ & $60.1 \pm 6.8$ & $22(2-78)$ \\
\hline Thyroid CA & 6 & 2.7 & $39.2 \pm 22.0$ & $45.6 \pm 18.2$ & $75(15-137)$ \\
\hline Pancreas CA & 4 & 1.8 & & & \\
\hline Uterus CA & 4 & 1.8 & & & \\
\hline Tongue CA & 4 & 1.8 & & & \\
\hline Prostate CA & 3 & 1.4 & & & \\
\hline Nasopharyngeal CA & 3 & 1.4 & & & \\
\hline Multiple myeloma & 2 & 0.9 & & & \\
\hline Others & 14 & 6.4 & & & \\
\hline Total & 220 & & & & \\
\hline
\end{tabular}

Values are expressed as mean \pm SD and median (range). $\mathrm{CA}=$ Carcinoma; NMSC = non-melanoma skin cancer; NHL = non-Hodgkin lymphoma. Bladder carcinoma include carcinoma of bladder and ureter.

\section{Results}

During the study period, dialysis was started in 6,574 patients. We excluded 320 patients with previous history of cancers, acquired immune deficiency syndrome or kidney transplantation prior to dialysis. As a result, 6,254 patients were analyzed. Among them, 3,403 (54.4\%) were male patients. The age at dialysis was $64.0 \pm 13.0$ (male $63.2 \pm 12.9$; female $64.9 \pm 13.1$ ). Seventy six percent were on PD while $24 \%$ were HD. The underlying causes of ESRD included diabetes mellitus (35\%), glomerulonephritis (25\%), hypertension (7\%), polycystic kidney disease (4\%), unknown (20\%) and others (9\%). After a mean follow-up of 2.4 years (range 2 months-20.1 years), 220 cancers were recorded in 220 patients. The overall incidence of cancers was $3.5 \%$. For those patients who developed cancers, the mean age at dialysis initiation was $59.3 \pm 14.6$, while the mean age at diagnosis of cancers was $63.9 \pm 12.5$. The mean interval between the dialysis initiation and the development of cancers was 4.5 years (range 2 months-27.3 years). The clinical characteristics of different common cancers (defined as occurrence in 5 or more cases in our cohort) were shown in table 1 . Colorectum was the most common site of cancer (20\%), followed by kidney (15.9\%), lung (13.6\%), breast (8.2\%) and liver (6.4\%).

The risks of any cancer occurrence were significantly higher in chronic dialysis patients than in the age-matched general population (SIR 1.44, 95\% CI 1.26-1.65). The SIRs of all cancers were then further analyzed according to the gender, age at dialysis, duration of follow-up and year of dialysis (table 2). The risk of cancers was higher in the female than in the male patients (1.76 vs. 1.24$)$. In addition, the risk of cancers was particularly high in patients younger than 45 years (the youngest age groups; SIR $40.48,95 \%$ CI 28.03-56.56), then decreased with age and finally became lower in patients older than 65 years (the oldest age group; SIR 0.81, 95\% CI 0.65-0.98), when compared with the general population. Furthermore, the cancer risk was higher in the first 2 years of dialysis, especially within the first year (SIR 8.10, 95\% CI 6.18-10.42). On the other hand, the cancer risk of the dialysis patients remained the same over time.

Table 3 showed the SIRs of the major cancer sites. The highest risk was observed for kidney cancer (SIR 12.28, 95\% CI 8.44-17.08), followed by cancers of tongue (SIR 4.21 , 95\% CI 1.13-10.78), cervix (SIR 4.12, 95\% CI 1.658.48), thyroid (SIR 3.42, 95\% CI 1.25-7.46), urinary blad- 
Table 2. Standardized incidence ratios for all malignancies, stratified by gender, age at dialysis, duration of follow-up and year of dialysis

\begin{tabular}{|c|c|c|c|}
\hline & Person-years & $\mathrm{O} / \mathrm{E}$ & SIR (95\% CI) \\
\hline All patients & 14,887 & $220 / 152.57$ & $1.44(1.26-1.65)$ \\
\hline \multicolumn{4}{|l|}{ Gender } \\
\hline Male & 7,342 & $113 / 91.36$ & $1.24(1.02-1.49)$ \\
\hline Female & 7,545 & $107 / 60.87$ & $1.76(1.44-2.12)$ \\
\hline \multicolumn{4}{|c|}{ Age at dialysis, years } \\
\hline$\leq 44$ & 916 & $34 / 0.84$ & $40.48(28.03-56.56)$ \\
\hline $45-64$ & 5,759 & $88 / 26.15$ & $3.37(2.70-4.15)$ \\
\hline$\geq 65$ & 8,212 & $98 / 121.65$ & $0.81(0.65-0.98)$ \\
\hline \multicolumn{4}{|c|}{ Duration of follow-up, years } \\
\hline$<1$ & 790 & $60 / 7.41$ & $8.10(6.18-10.42)$ \\
\hline $1-2$ & 1,926 & $38 / 18.06$ & $2.10(1.49-2.89)$ \\
\hline $2-5$ & 6,592 & $59 / 67.85$ & $0.87(0.66-1.12)$ \\
\hline $5-10$ & 4,412 & $48 / 44.49$ & $1.08(0.80-1.43)$ \\
\hline$\geq 10$ & 1,167 & $15 / 10.84$ & $1.38(0.77-2.28)$ \\
\hline \multicolumn{4}{|l|}{ Year of dialysis } \\
\hline Before 2000 & 3,723 & $58 / 38.16$ & $1.52(1.15-1.96)$ \\
\hline 2000-2009 & 7,988 & $113 / 81.86$ & $1.38(1.14-1.66)$ \\
\hline 2010-2014 & 3,176 & $49 / 32.55$ & $1.51(1.11-1.99)$ \\
\hline
\end{tabular}

$\mathrm{O}=$ Observed number of cancer cases; $\mathrm{E}=$ expected number of cancer cases, based on the general population in Hong Kong, from Hong Kong Cancer Registry.

Table 3. Site-specific cancer risk in chronic dialysis patients

\begin{tabular}{lcc}
\hline Site of cancer & O/E & SIR $(95 \% \mathrm{CI})$ \\
\hline Kidney & $35 / 2.85$ & $12.28(8.44-17.08)$ \\
Tongue & $4 / 0.95$ & $4.21(1.13-10.78)$ \\
Cervix & $7 / 1.70$ & $4.12(1.65-8.48)$ \\
Thyroid & $6 / 1.75$ & $3.43(1.25-7.46)$ \\
Bladder & $9 / 3.60$ & $2.50(1.14-4.75)$ \\
NMSC & $8 / 4.58$ & $1.75(0.75-3.44)$ \\
NHL & $7 / 4.15$ & $1.69(0.68-3.48)$ \\
Uterus & $4 / 2.43$ & $1.65(0.44-4.21)$ \\
Breast & $18 / 10.94$ & $1.65(0.97-2.60)$ \\
Colorectal & $44 / 28.77$ & $1.53(1.11-2.05)$ \\
Multiple myeloma & $2 / 1.53$ & $1.31(0.15-4.72)$ \\
Liver & $14 / 11.23$ & $1.25(0.68-2.09)$ \\
Pancreas & $4 / 3.43$ & $1.17(0.31-2.99)$ \\
Stomach & $8 / 7.28$ & $1.10(0.47-2.17)$ \\
Nasopharynx & $3 / 3.09$ & $0.97(0.2-2.84)$ \\
Lung & $30 / 31.96$ & $0.94(0.63-1.34)$ \\
Prostate & $3 / 11.1$ & $0.27(0.05-0.79)$ \\
\hline
\end{tabular}

$\mathrm{O}=$ Observed number of cancer cases; $\mathrm{E}=$ expected number of cancer cases, based on the general population in Hong Kong, from Hong Kong Cancer Registry.
Table 4. SIRs for kidney cancer, stratified by gender, age at dialysis and duration of follow-up

\begin{tabular}{|c|c|c|}
\hline & Number & $\operatorname{SIR}(95 \% \mathrm{CI})$ \\
\hline All patients & 35 & $12.28(8.44-17.08)$ \\
\hline \multicolumn{3}{|l|}{ Gender } \\
\hline Male & 13 & $9.77(5.20-16.72)$ \\
\hline Female & 22 & $16.06(10.06-24.31)$ \\
\hline \multicolumn{3}{|c|}{ Age at dialysis, years } \\
\hline$\leq 44$ & 11 & $550.00(274.18-984.17)$ \\
\hline $45-64$ & 17 & $30.14(17.55-48.26)$ \\
\hline$\geq 65$ & 7 & $3.30(1.32-6.81)$ \\
\hline \multicolumn{3}{|c|}{ Duration of follow-up, years } \\
\hline$<1$ & 7 & $50.00(20.03-103.02)$ \\
\hline $1-2$ & 3 & $8.57(1.72-25.04)$ \\
\hline $2-5$ & 4 & $3.33(0.90-8.53)$ \\
\hline $5-10$ & 10 & $12.50(5.98-22.99)$ \\
\hline$\geq 10$ & 11 & $51.89(25.87-92.85)$ \\
\hline
\end{tabular}

der (SIR 2.5, 95\% CI 1.14-4.75) and colorectum (SIR 1.53, 95\% CI 1.11-2.05). On the other hand, the risk of prostate cancer was lower (SIR 0.27, 95\% CI 0.05-0.79) in the dialysis patients. For kidney cancer, the risk was higher in female than in male patients, and in younger than older patients. In addition, the duration of dialysis seemed to influence the relative risk of kidney cancer as well (table 4).

Totally 160 dialysis patients with cancer $(72.7 \%)$ died during the follow-up period. Among them, 89 were males and 71 were females. The mean age was $68.5 \pm 10.3$ at the time of death. The mean survival time after diagnosis of cancers was 25.5 months (range 0-275 months). The main cause of death was cancer progression $(50.6 \%, \mathrm{n}=$ $81)$, followed by infection $(25.6 \%, n=41)$. Other recognized causes included ischemic heart disease and cerebrovascular accident.

The cancer mortality risk was similar in the dialysis patients when compared to the general population (SMR 0.91, 95\% CI 0.72-1.13). The SMRs of all cancers were further analyzed according to gender and age at dialysis. The SMR of male and female patients was 1.07 (95\% CI 0.79-1.42) and 0.75 (95\% CI 0.52-1.05) respectively. On the other hand, the cancer mortality risk was the highest in young patients and then it decreased with age (SMR 10.92, 95\% CI $1.23-39.42$ for age $\leq 44$ at dialysis and SMR 2.34, 95\% CI 1.50-3.48 for age in the range 45-64 at dialysis). For patients who started dialysis at age $\geq 65$, the risk of cancer death was lower than those in the general population (SMR 0.70, 95\% CI $0.53-0.91)$. 
The more common causes of cancer death included lung cancer ( $\mathrm{n}=17$, SMR $0.63,95 \%$ CI $0.37-1.01)$, colorectal cancer $(\mathrm{n}=15$, SMR $1.11,95 \%$ CI $0.62-1.82)$, liver cancer ( $\mathrm{n}=7$, SMR $0.69,95 \%$ CI 0.28-1.43), kidney cancer ( $\mathrm{n}=6$, SMR 4.92, 95\% CI 1.80-10.70), stomach cancer $(\mathrm{n}=5$, SMR 1.11, 95\% CI 0.36-2.58) and bladder cancer $(\mathrm{n}=4$, SMR 2.74, 95\% CI 0.74-7.01).

\section{Discussion}

In accordance with the previous findings in literature, the chronic dialysis patients in our study also had an elevated risk of cancer when compared with the general population. In our study, the SIR was 1.44 (95\% CI 1.261.65), which was comparable to the Taiwanese study (SIR $1.4,95 \%$ CI 1.3-1.4) [12]. In addition, $3.5 \%$ of the dialysis patients had cancers, a figure which was also similar to Australia (3.7\%), the United States (3.4\%) and Europe (2.3\%) [3].

The SIR of overall cancers in the female dialysis patients was higher than that in the male dialysis patients, which was consistent with other registry studies $[3,17]$. In the international collaborative study, the excessive cancer risk was found to be the highest in the younger dialysis patients, gradually declined with increasing age and became only slightly elevated in the dialysis patients within the oldest age group in all 3 registry population [3]. The higher excess in the youngest age group was probably related to the low background cancer rates among the younger individuals in the general population. Our study also showed a similar trend except that the elderly dialysis patients ( $\geq 65$ years) had an even lower risk of overall cancers when compared with the general population. Indeed, such finding was also shown in the Taiwanese study [17]. The expected survival of the elderly dialysis patients could be significantly reduced by the presence of ESKD, significant comorbidities and frailty. As a result, these patients may die earlier, probably due to cardiovascular diseases, cerebrovascular events or infections, before the development of cancers. In addition, due to the lower life expectancy and comorbidities, the elderly dialysis patients are less likely to receive cancer screening or invasive diagnostic procedures than the elderly in the general population. The consequence is that cancers could be underestimated in the elderly patients suffering from ESKD, especially those with poor function status.

Our study also showed that the highest cancer risks occurred early after starting dialysis, especially in the first year. This is in agreement with other studies $[3,17]$. A possible explanation was the presence of detection bias, as a result of higher incidence of recognized cancers with closer surveillance in patients suffering from ESKD.

In general, cancers in patients suffering from ESKD can be classified according to whether they are related to ESKD or immune deficiency $[6,7]$. Cancers are categorized as ESKD-related when a more than 2-fold risk in patients with ESKD prior to starting dialysis with no associated or smaller excess risk in persons with HIV infection. All other cancers are categorized as being or not being related to immune deficiency if the cancer involved is increased or not increased in persons with HIV infection as well as in transplant recipients [20]. Cancers of kidney, urinary tract and thyroid are classified as ESKD-related malignancy because the risk is as high in patients with ESKD (before or after dialysis) as it is after transplantation [6].

Similar to the Australia and New Zealand Registry data, the highest risk in the dialysis patients in our study was kidney cancer, which is a well-known consequence of acquired cystic kidney disease (ACKD). The cancer risk was particularly high within the first year of dialysis, probably due to the closer surveillance and detection bias as explained above. In addition, the cancer risk also showed an increasing trend with the duration of dialysis. This was compatible with the report from different studies that long-term dialysis therapy could be associated with a higher incidence of kidney cancer in ESKD patients $[20,21]$. Current literature shows that most patients with ESKD will develop acquired renal cysts after 3-7 years on dialysis, associated with a 1.6-7\% incidence of renal cell carcinoma (RCC) $[22,23]$. Whether RCC is also increased by immune deficiency is not yet certain. Several factors were reported to cause RCC in chronic dialysis patients with ACKD. Increased expression of growth factors or uremic metabolites has been demonstrated in patients with ESKD. Stimulation of tubular and cystic cells by these substances may promote the development of ACKD, with progressively more florid degrees of papillary hyperplasia and papillary cancer development either simultaneously or subsequently [24].

Some diseases that cause ESKD are also risk factors for urinary tract cancers. Among them, analgesic nephropathy is a common example. In a study of chronic HD patients in China, transitional cell carcinoma (TCC) of the urinary tract was the most common cancer. Most of these patients with TCC had aristolochic acid nephropathy as the underlying disease [25]. In the Taiwanese study, the urinary bladder was also the most common site of cancer development in the dialysis patients (21.2\%) with an SIR 
of 8.2 (95\% CI 6.7-9.9) [17]. However, TCC of urinary bladder ranked only as the sixth commonest cancer (4.1\%) in our study and the SIR was 2.50 (95\% CI 1.144.75).

The incidence of thyroid cancer was found to be higher in both dialysis patients and kidney transplant recipients when compared with the general population $[3,6]$. The SIR was 3.43 (95\% CI 1.25-7.46) in the dialysis patients in our study. Our previous study also confirmed a higher risk in kidney transplant recipients (SIR 4.35, 95\% CI 2.41-7.85) [26]. The exact mechanism for the increased risk is still unclear. Since there is no increase of thyroid cancer in patients with HIV infection, contribution from immune deficiency is less likely $[6,7,20]$.

In agreement with the Australia and New Zealand Registry data [6], higher risks of tongue cancer and cervical cancer in dialysis patients were also observed in our study. Both cancers are known to be associated with human papillomavirus (HPV) and it has been postulated that uremic immune dysfunction, supported by the evidence of reactivation of latent Epstein-Barr virus infection in uremic immunodeficiency [27] could be a possible cause. In addition, the use of immunosuppressive agents during treatment of the underlying diseases leading to ESKD could also result in a higher risk of these HPV-related cancers.

Another result worth mentioning about our study is that there was a markedly lower risk of prostate cancer in dialysis patients. Indeed, similar findings were also observed in other studies $[3,6,28]$. This inverse association may be a manifestation of screening. Prostate-specific antigen (PSA) is a commonly used tool for prostate cancer screening in the general population. In the United States, approximately $40 \%$ of prostate cancer cases are detected through PSA testing [29]. Due to the lower life expectancy, men with ESKD may not receive screening with the PSA test as frequently as other men, leading to an apparent lower incidence of prostate cancer.

The overall risk of cancer death in the dialysis patients was comparable to the age- and sex-matched population in our study. In fact, a higher SMR was observed only in younger patients, while among the older patients, the
There were some limitations to our study. First, it was a retrospective study and various risk factors of cancers such as smoking, alcohol consumption and family history of cancers were not recorded. Moreover, since only 4 renal centers participated in this study, the possibility of selection bias should still be considered although the clinical characteristics of our cohort and the dialysis population in Hong Kong were similar [30]. Furthermore, the size of our cohort was small when compared with other registries in the United States, Europe, Australia or New Zealand. There is insufficient data to provide gender-specific estimates of each cancer from this study. However, the data on dialysis obtained from our renal registry and patients' own medical records are complete and accurate. In addition, the cancer data, including both incidence and mortality, obtained from the Hong Kong Cancer Registry are also of high standard. The percentage of histologically verified cases and death certificate only cases (the 2 key performance indicators of data completeness) was 87 and $0.7 \%$, respectively. This is already up to the highest International Association of Cancer Registries standard for developed countries [19].

In conclusion, we can confirm that the incidence of cancers in the Chinese chronic dialysis patients, especially the young patients, was elevated when compared with the general population. Moreover, the cancer mortality risk was highest in young patients and then decreased with age, probably due to the potential competing risk of cardiovascular mortality in older dialysis patients. Among the different cancers, only kidney cancer had an elevated SMR. Currently, there is no active cancer screening in ESKD patients. However, our findings of substantially increased risks in young patients, particularly in relation to kidney cancer, indicate that we can adopt a more individualized approach to cancer screening in chronic dialysis patients.

\section{Disclosure Statement}

None. Chak/Chau/Lui/Lo/Tang overall SMR was much lower than that in the general population. This can be explained by the potential competing risk of death from cardiovascular disease in older patients, which may dampen the effect of dialysis on cancer mortality risk in the chronic dialysis patients. Among the different cancers identified in our cohort, only those with kidney cancer had higher cancer mortality than the general population (SMR 4.92, 95\% CI 1.80-10.70).

References

Cheung/Chan/Chan/Ng/Lam/Wong/

1 Kinlen LJ, Eastwood JB, Kerr DN, et al: Cancer in patients receiving dialysis. Br Med J 1980;280:1401-1403.

2 Lindner A, Farewell VT, Sherrard DJ: High incidence of neoplasia in uremic patients receiving long-term dialysis. Cancer and longterm dialysis. Nephron 1981;27:292-296.

3 Maisonneuve P, Agodoa L, Gellert R, et al: Cancer in patients on dialysis for end-stage renal disease: an international collaborative study. Lancet 1999;354:93-99. 
4 Matas AJ, Simmons RL, Kjellstrand CM, Buselmeier TJ, Najarian JS: Increased incidence of malignancy during chronic renal failure. Lancet 1975;1:883-886.

5 Curtis JR: Cancer and patients with end-stage renal failure. Br Med J (Clin Res Ed) 1982;284: 69-70.

6 Vajdic CM, McDonald SP, McCredie MR, et al: Cancer incidence before and after kidney transplantation. JAMA 2006;296:2823-2831.

7 Stewart JH, Vajdic CM, van Leeuwen MT, Amin J, Webster AC, Chapman JR, McDonald SP, Grulich AE, McCredie MR: The pattern of excess cancer in dialysis and transplantation. Nephrol Dial Transplant 2009;24 3225-3231.

8 Butler AM, Olshan AF, Kshirsagar AV, et al: Cancer incidence among US Medicare ESRD patients receiving hemodialysis, 1996-2009. Am J Kidney Dis 2015;65:763-772.

9 Wong G, Hayen A, Chapman JR, et al: Association of CKD and cancer risk in older people. J Am Soc Nephrol 2009;20:1341-1350.

10 Vogelzang JL, van Stralen KJ, Noordzij M, et al: Mortality from infections and malignancies in patients treated with renal replacement therapy: data from the ERA-EDTA registry. Nephrol Dial Transplant 2015;30:1028-1037.

11 Wakasugi M, Kazama JJ, Yamamoto S, Kawamura K, Narita I: Cause-specific excess mortality among dialysis patients: comparison with the general population in Japan. Ther Apher Dial 2013;17:298-304.

12 Mandayam S, Shahinian VB: Are chronic dialysis patients at increased risk for cancer? J Nephrol 2008;21:166-174.

13 Vamvakas S, Bahner U, Heidland A: Cancer in end-stage renal disease: potential factors involved. Am J Nephrol 1998;18:89-95.
14 Lee JE, Han SH, Cho BC, et al: Cancer in patients on chronic dialysis in Korea. J Korean Med Sci 2009;24(suppl):S95-S101.

15 Ota K, Yamashita N, Suzuki T, Agishi T: Malignant tumours in dialysis patients: a nationwide survey. Proc Eur Dial Transplant Assoc 1981;18:724-730.

16 Loy EY, Choong HL, Chow KY: Cancer among end-stage renal disease patients on dialysis. Ann Acad Med Singapore 2013;42: 640-645.

17 Lin HF, Li YH, Wang CH, Chou CL, Kuo DJ, Fang TC: Increased risk of cancer in chronic dialysis patients: a population-based cohort study in Taiwan. Nephrol Dial Transplant 2012;27:1585-1590.

18 Leung CB, Cheung WL, Li PK: Renal registry in Hong Kong-the first 20 years. Kidney Int Suppl (2011) 2015;5:33-38.

19 Hospital Authority: Hong Kong Cancer Registry Web Site.http://www3.ha.org.hk/cancereg/ statistics.html (accessed October 2015).

20 Grulich AE, van Leeuwen MT, Falster MO, Vajdic CM: Incidence of cancers in people with HIV/AIDS compared with immunosuppressed transplant recipients: a meta-analysis. Lancet 2007;370:59-67.

21 Stewart JH, Buccianti G, Agodoa L, Gellert R, McCredie MR, Lowenfels AB, Disney AP, Wolfe RA, Boyle P, Maisonneuve P: Cancers of the kidney and urinary tract in patients on dialysis for end-stage renal disease: analysis of data from the United States, Europe, and Australia and New Zealand. J Am Soc Nephrol 2003; 14:197-207.
22 Ishikawa I: Acquired cystic disease: mechanisms and manifestations. Semin Nephrol 1991;11:671-684.

23 Levine E, Slusher SL, Grantham JJ, Wetzel LH: Natural history of acquired renal cystic disease in dialysis patients: a prospective longitudinal CT study. AJR Am J Roentgenol 1991;156:501-506.

24 Hughson MD, Schmidt L, Zbar B, Daugherty S, Meloni AM, Silva FG, Sandberg AA: Renal cell carcinoma of end-stage renal disease: a histopathologic and molecular genetic study. J Am Soc Nephrol 1996;7:2461-2468.

25 Zhou L, Cao YL, Li WG, Fu FT, Zhang L, Wang X, Shi XH: Transitional cell carcinoma associated with aristolochic acid nephropathy: most common cancer in chronic hemodialysis patients in China. Chin Med J (Engl) 2012;125:4460-4465.

26 Cheung CY, Lam MF, Chu KH, et al: Malignancies after kidney transplantation: Hong Kong renal registry. Am J Transplant 2012;12: 3039-3046.

27 Winkelspecht B, Mueller-Lantzsch N, Köhler $\mathrm{H}$ : Serological evidence for reactivation of EBV infection due to uraemic immunodeficiency. Nephrol Dial Transplant 1997;12: 2099-2104.

28 Shebl FM, Warren JL, Eggers PW, Engels EA: Cancer risk among elderly persons with endstage renal disease: a population-based casecontrol study. BMC Nephrol 2012;13:65.

29 Draisma G, Etzioni R, Tsodikov A, et al: Lead time and overdiagnosis in prostate-specific antigen screening: importance of methods and context. J Natl Cancer Inst 2009;101:374-383.

30 Ho YW, Chau KF, Choy BY, et al: Hong Kong renal registry report 2012. Hong Kong J Nephrol 2013;15:28-43. 